\title{
Presentation of the CALMAR-ECORCES spectrum adjustment package available at the NEA Data bank
}

\author{
Gilles Grégoire* \\ CEA, DEN, DER/SPEX, F-13108 Saint-Paul-lez-Durance, France \\ Christophe Destouches, Clément Fausser, and Nicolas Thiollay
}

\begin{abstract}
Adjustment methods are well known for the determination of a multigroup spectrum from foils activity measurements [1, 2] and have been included for many years in ASTM standards [3]. These inversion methods combine information from activity measurements of foils with their respective response functions (cross sections and covariances) to provide a best-estimate multigroup spectrum. According to the selected method, one should provide an optional prior spectrum as input. An adjustment library CALMAR has been developed and some aspects of the library has already been exposed [4, this paper focusses on the public release of the library. It describes the ECORCES module for nuclear data preparation and CALMAR module for the adjustment procedure. Short samples of validation are shown.
\end{abstract}

\section{INTRODUCTION}

The IRDFF dosimetry library [5] is under constant development but specific dosimetry tools for extracting cross section such as X333 [6] are not maintained any more. Cross section processing before adjustment now relies on generalist tools such as NJOY[10], designed for evaluators. So a new package containing an a cross section processing tool (ECORCES) mainly dedicated to IRDFF and an adjustment code (CALMAR) has been developed and qualified by the CEA. This paper describes the main package's features and presents elements of its validation. Finally, information on the coming package publication at the OCDE/NEA code data bank are given.

\footnotetext{
* gilles.gregoire@cea.fr
} 


\section{ECORCES UTILITY FOR CROSS SECTION EXTRACTION}

The ECORCES code proposes the following functions:

- Extraction of cross sections and associated covariance matrices from the IRDFF and IRDF2002 nuclear data files (pointwise or multi-group format);

- Calculation of the multi-group cross sections, spectrum and covariance or correlation matrices in a new selected energy grid. Data are condensed with a neutron spectrum and an energy grid given by the user.

- Calculation of the cumulated reaction rates, cross sections and spectrum level with the associated uncertainties.

Basically, the ECORCES main principle is the conservation of reaction rates from initial grid $\left(G_{i n}\right)$ computed in the new grid $\left(G_{\text {out }}\right)$.

$$
\int_{G_{\text {in }}} \sigma(E) \phi(E) d E=\int_{G_{\text {out }}} \sigma(E) \phi(E) d E
$$

\section{Algorithm for computing section or flux in a new energy grid}

We define the three grids needed in computation:

- $G_{\sigma}$ : grid of the cross section in IRDFF library

- $G_{\phi}$ : grid of input flux

- $G_{\text {out }}$ : output user grid

The cross sections and the input flux are computed on the union grid defined by:

$$
G_{\cup}=G_{\sigma} \cup G_{\phi} \cup G_{\text {out }}
$$

The new inserted points on the union grid $G_{\cup}$ are computed using interpolation laws. The second part of the computation consists in collapsing previously computed data on union grid into the user grid.

This computation is done for each group $g_{\text {out }}$ of the output grid $G_{\text {gout }}$, using data from groups $g_{\cup}$ of the union grid $G_{\cup}$ with the equation 3 ;

$$
\sigma_{g_{\text {out }} \in G_{\text {out }}}=\frac{\sum_{g \cup g_{\text {out }}} \int_{g_{\cup} \in G_{\text {out }}} \sigma(E) \phi(E) d E}{\sum_{g \cup \subset g_{\text {out }}} \int_{g_{\cup} \in G_{\text {out }}} \phi(E) d E}
$$




\section{Algorithm for computing covariance in a new grid}

The reaction rate uncertainty $\Gamma_{\tau}$, when neglecting the cross covariance term, contains two terms:

$$
\Gamma_{\tau}=\operatorname{Var}(\Sigma \phi)=\Sigma \Gamma_{\phi} \Sigma^{T}+\phi^{T} \Gamma_{\Sigma} \phi
$$

The reaction rate uncertainty can be simplified using relative covariances (reduced variables):

$$
\Gamma_{\tau}=\Sigma \phi^{T} \Gamma_{\phi}^{R} \Sigma^{T} \phi+\phi^{T} \Sigma \Gamma_{\Sigma}^{R} \Sigma^{T} \phi=\operatorname{diag}(\tau)\left[\Gamma_{\phi}^{R}+\Gamma_{\Sigma}^{R}\right] \operatorname{diag}(\tau)
$$

The transformation of reaction rates from union grid to output grid can be written as a matrix product and transformed with reduced variables:

$$
\tau_{G_{\text {out }}}=P \tau_{G_{\cup}} \Leftrightarrow \mathbf{1}_{G_{\text {out }}}=P^{R} \mathbf{1}_{G_{\cup}}
$$

The $P^{R}$ is the transfer matrix for relative covariance using the sandwich rule (equation 7):

$$
\Gamma_{\tau_{G_{\text {out }}}^{R}}^{R}=P^{R}\left[\Gamma_{\phi}^{R}+\Gamma_{\Sigma}^{R}\right]\left(P^{R}\right)^{T}
$$

The covariance computation algorithm first needs cross section and flux computed in the union grid: the covariance matrix is generated in the union grid from the NI sub-subsections. The algorithm constructs the $P^{R}$ transfer matrix between the union grid and the output grid (size of $G_{\text {out }}$ rows and size of $G_{\cup}$ columns).

\section{Validation against existing tools}

ECORCES modules has been validated on the computation of averaged cross sections on reference spectra and has been compared to codes such as INTER (https://www-nds. iaea.org/public/endf/utility/) or RR_UNC (https://www-nds.iaea.org/IRDFF/).

Table 1 shows some values computed on the Maxwell spectrum provided with IRDFF 1.05. discrepancies are very small and mainly due to numerical precision treatment. All values with different types of IRDFF spectra are available in the package as test cases. 


\begin{tabular}{|c|c|c|}
\hline Tool & ECORCES & RR_UNC \\
\hline Reaction & $\sigma(b)$ & $\sigma(b)$ \\
\hline${ }^{59} \mathrm{Co}(n, \gamma)$ & 37.1925 & 37.19 \\
\hline${ }^{197} A u(n, \gamma)$ & 99.1808 & 99.175 \\
\hline${ }^{45} S c(n, \gamma)$ & 27.2142 & 27.213 \\
\hline
\end{tabular}

TABLE 1: Validation of ECORCES against RR_UNC utility: computation of Maxwell spectrum averaged cross section

\section{CALMAR ADJUSTMENT MODULE}

The CALMAR module is a least square based adjustment program[4] as STAYS'L[1]. In addition to STAYS'L, an automatic neutron spectrum level adjustment is computed.

\section{Algorithm}

The process of adjustment is described by these following steps:

1. Transform all inputs to reduced variables to enhance matrix conditioning. All following computations are done on reduced variables.

2. C scaling factor is determined by minimizing weighted quadratic difference between measured $\left(\tau^{m}\right)$ and computed $\left(\tau^{c}\right)$ rates (8) where $W=\left[\Gamma_{m}+C^{2} \Gamma_{\tau^{c}}\right]^{-1}$.

$$
\chi_{\text {min }}^{2}=\left(\tau^{m}-C \tau^{0}\right)^{T} W\left(\tau^{m}-C \tau^{0}\right)
$$

3. Computing shape $\hat{\phi}$ with least square formula (9), using the scaled spectrum.

$$
\hat{\phi}=\phi^{0}+C \Gamma_{\phi^{0}} \Sigma W\left(\tau^{m}-C \tau^{0}\right)
$$

4. Update weighting matrix.

5. End loop when convergence criteria $\left(<10^{-5}\right)$ is reached on $C$. Only few iterations are necessary.

6. Compute final values and uncertainties with the conditional expectation formalism (Schur complement or inversion lemma). The covariance of scaled adjusted spectrum is deduced from higher-order moments formula for multivariate distributions [7]. 


\section{Effect of scaling on results}

This section shows on figure the combined effects of shape and level on results. The second graph on Figure 1 shows the multigroup adjustment ratio. Below $1 \mathrm{MeV}$, this ratio is constant: only one foil gives information to the process and this information is used for scaling. Above $1 \mathrm{MeV}$, ratio is variable and not monotone: many foils give information on specific part of the spectrum.
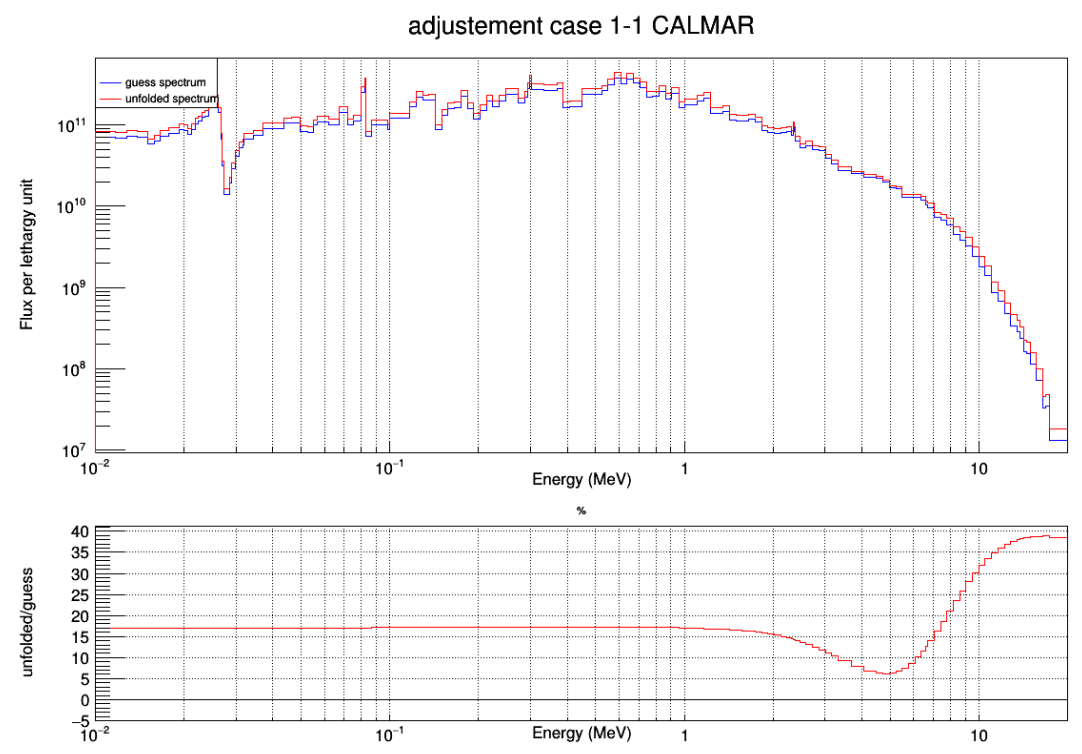

FIG. 1: Guess and adjusted multigroup fluxes and multigroup ratio

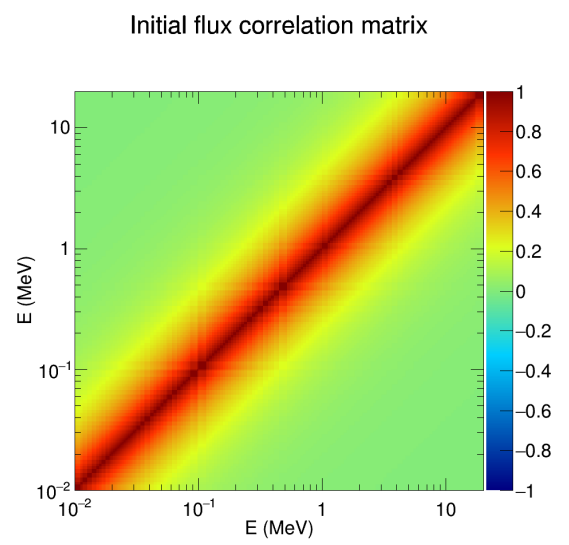

(a) guess covariance

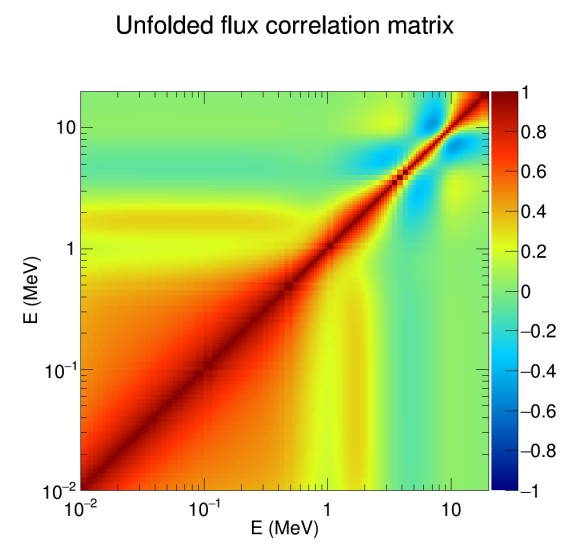

(b) adjusted covariance

FIG. 2: Covariance outputs of CALMAR

Guess and adjusted multigroup covariances are shown on figure 2. Guess flux covariance 
has been constructed as a short range covariance (continous, no structures). After adjustment, groups below $1 \mathrm{MeV}$ are more correlated, due to the fact there is only one foil that gives information. This information corresponds to flux scaling. On the contrary, above $1 \mathrm{MeV}$, information is brought by foils and initial correlation is reduced.

\section{Validation of CALMAR}

CALMAR has been validated against existing codes such as STAYS'L, MSITER 9], which are based on least-squares formalism. Table 2 shows results for fast flux $(>1 \mathrm{MeV})$ comparison. Considering these results, STAYS'L without scaling can not attain expected value because it is too constrained. Manual scaling with STAYS'L shows a better performance but uncertainty computation is limited. MSITER and CALMAR results are quite similar. In the treatment of uncertainties, part of experimental uncertainty used during scaling step is correctly taken into account in final steps: the final uncertainty is somewhat bigger than for STAYS'L code.

\begin{tabular}{|c|c|c|c|c|}
\hline Codes & $\begin{array}{c}\text { Scaling } \\
\text { method }\end{array}$ & $\begin{array}{c}\text { Flux }>1 \mathrm{MeV} \\
\times 10^{11} n . \mathrm{cm}^{-2} . \mathrm{s}^{-1}\end{array}$ & $\begin{array}{c}\text { Unc. } \% \\
(k=1)\end{array}$ & $\begin{array}{c}\text { Code } \\
\text { Reference }\end{array}$ \\
\hline Guess flux & & 1.43 & 10.3 & \\
\hline STAYS'L & no & 1.58 & 4.4 & {$[1]$} \\
\hline STAYS'L & manual & 1.64 & 4.6 & {$[1]$} \\
\hline MSITER & auto. & 1.65 & 5.2 & {$[9]$} \\
\hline CALMAR & auto. & 1.65 & 5.2 & this paper \\
\hline
\end{tabular}

TABLE 2: Validation of CALMAR against existing tools

\section{TYPICAL USE OF ECORCES AND CALMAR TOOLS}

Modules have been developed to benefit from all ROOT capabilities. The typical use of are described in table 3 . This is a guide to help the user to choose the best method of using the package, depending on project type, programming skills...

As input for codes, dosimetry libraries (IRDF2002 [8] and IRDFF 1.05 [5]) are provided 
with the package. It also contains IRDFF reference spectra (Maxwell, 1/E, Fission,...) needed to compute integral cross sections.

\begin{tabular}{|c|c|c|c|c|c|}
\hline Method & Skills & Inputs & Outputs & Automation & Typical use \\
\hline $\begin{array}{l}\text { standalone tools } \\
\text { ecorces } \\
\text { sepiida } \\
\text { (calmar standalone) }\end{array}$ & linux & $\begin{array}{l}\text { flat files, } \\
\text { IRDFF library }\end{array}$ & $\begin{array}{l}\text { flat files, } \\
\text { tool defined, } \\
\text { no wrappers } \\
\text { basic graphs }\end{array}$ & shell tools & $\begin{array}{l}\text { basic usage, } \\
\mathrm{C}++ \text { not required }\end{array}$ \\
\hline $\begin{array}{l}\mathbf{C}++ \text { classes } \\
\text { ROOT script }\end{array}$ & $\begin{array}{l}\mathrm{C}++ \\
\mathrm{ROOT}\end{array}$ & $\begin{array}{l}\text { flat files, } \\
\text { IRDFF library, } \\
\text { user defined } \\
\text { (ROOT files) }\end{array}$ & $\begin{array}{l}\text { flat files, } \\
\text { user defined } \\
\text { via classes } \\
\text { interfaces, } \\
\text { ROOT graphs }\end{array}$ & $\begin{array}{l}\text { ROOT } \\
\text { interpreter }\end{array}$ & $\begin{array}{l}\text { simple automation, } \\
\text { lightweight code } \\
\text { coupling }\end{array}$ \\
\hline $\begin{array}{l}\mathbf{C}++ \text { classes } \\
\text { library }\end{array}$ & $\begin{array}{l}\mathrm{C}++, \\
\text { ROOT, } \\
\text { build env. }\end{array}$ & $\begin{array}{l}\text { user defined, } \\
\text { data from } \\
\text { calling code }\end{array}$ & $\begin{array}{l}\text { user defined } \\
\text { via classes } \\
\text { interfaces, } \\
\text { ROOT graphs }\end{array}$ & $\begin{array}{l}\text { calling } \\
\text { function }\end{array}$ & $\begin{array}{l}\text { complex automation, } \\
\text { full code coupling }\end{array}$ \\
\hline
\end{tabular}

TABLE 3: Usage of the package : reference table

\section{NEA AVAILABILITY OF THE CODE}

The release of the package at the NEA databank was motivated by giving to the dosimetry community an updated adjustment package based on the newest IRDFF library. Last package released with IRDF was NMF90[9], more than twenty year ago and processing tools are not maintained any more. The package needs only ROOT framework but does not rely on other codes such as NJOY [10]. To avoid incompatibilities during installation, an open-source library licence, CeCILL-C (www.cecill.info) has been chosen. Open-source licence ensure that methods in the code are kept available to the community. $\mathrm{C}++[11]$ programming gives modularity and extendibility. 


\section{Contents of the package}

In the package, one can find :

- $\mathrm{C}++$ source code

- IRDFF library

- reference manual

- test cases

In the reference manual, installation procedure is included to generate binaries from source code. Automatic test scripts are also included. HTML interactive documentation of the $\mathrm{C}++$ classes can be generated from sources with doxygen tool (http://doxygen.org).

\section{CONCLUSION}

A new adjustment package, ECORCES and CALMAR, will be available in 2017 at NEA data bank more than twenty years after NMF-90. This module differs from existing code by integrating all needed tools in a same package: extraction tool from libraries and adjustment module. The package has been validated against existing codes and data. It has been made modular and versatile such as every user can find a suitable execution mode for its own application, simple or complex problem.

[1] F. Perey, "Least-square dosimetry unfolding: the program STAY'SL," Tech. Rep. TM-6062 ENDF-254, ORNL, 1977.

[2] W. McElroy, S. Berg, T. Crockett, and R. Hawkins, "A computer automated iterative method for neutron flux spectra determination by foil activation," Tech. Rep. AFWL-TR-67-41-Vol1, Air Force Weapons Laboratory, Kirtland, NM, 1967.

[3] ASTM-E944, "E944-13 standard guide for application of neutron spectrum adjustment methods in reactor surveillance E706-IIA," West Conshohocken, PA: ASTM International, 2013. 
[4] Grégoire, G. and al, "CALMAR: A new versatile code library for adjustment from measurements," in $15^{\text {th }}$ Int. Symp. on Reactor Dosimetry, vol. 106, (Aix en Provence, France), p. 07006, 2016.

[5] E. Zsolnay, R. C. Noy, H. Nolthenius, and A. Trkov, "The International Reactor Dosimetry and Fusion File (IRDFF release 1.02)," Tech. Rep. IAEA-NDS-0616, IAEA, 2012.

[6] E. Szondi and H. Nolthenius, "User's guide to the cross section processing code X333," Tech. Rep. BME-NTI 222/95, Institution of Nuclear Techniques, Technical University of Budapest, May 1995.

[7] L. Isserlis, "On a formula for the product-moment coefficient of any order of a normal frequency distribution in any number of variables," Biometrika, vol. 12, no. 1-2, pp. 134-139, 1918.

[8] IAEA, "The International Reactor Dosimetry File 2002 (IRDF2002)," Tech. Rep. IAEA-NDS452, IAEA, 2002.

[9] N. Kocherov, "Neutron metrology file 90 - NMF90, an integrated database for performing neutron spectrum adjustment calculation," Tech. Rep. INDC-NDS-347, IAEA, January 1996.

[10] D. Muir, R. Boicourt, and A. Kahler, "the the NJOY Nuclear Data Processing System, version 2012,” Tech. Rep. LA-UR-12-27079, Los Alamos National Laboratory, December 2012.

[11] ISO/IEC, "Programming languages, C++,ISO/IEC 14882:2003," 2003. 